\title{
Mass spectrometry based metabolomics approach on the elucidation of volatile metabolites formation in fermented foods: A mini review
}

\author{
Min Kyung Park ${ }^{1}$ Young-Suk Kim ${ }^{1}$ (1)
}

Received: 14 February 2021/Revised: 12 April 2021/Accepted: 3 May 2021 / Published online: 8 July 2021

(c) The Author(s) 2021

\begin{abstract}
Metabolomics can be applied for comparative and quantitative analyses of the metabolic changes induced by microorganisms during fermentation. In particular, mass spectrometry (MS) is a powerful tool for metabolomics that is widely used for elucidating biomarkers and patterns of metabolic changes. Fermentation involves the production of volatile metabolites via diverse and complex metabolic pathways by the activities of microbial enzymes. These metabolites can greatly affect the organoleptic properties of fermented foods. This review provides an overview of the MS-based metabolomics techniques applied in studies of fermented foods, and the major metabolic pathways and metabolites (e.g., sugars, amino acids, and fatty acids) derived from their metabolism. In addition, we suggest an efficient tool for understanding the metabolic patterns and for identifying novel markers in fermented foods.
\end{abstract}

Keywords Metabolomics - Mass spectrometry ·

Fermentation - Volatile metabolites - Metabolic pathway

\section{Mass spectrometry-based metabolomics strategies}

Omics tools can be used as a platform to provide strategies for controlling and changing the qualities of fermented foods. The various types of omics can be divided according to the target compounds, such as genes (genomics), mRNA

Young-Suk Kim

yskim10@ewha.ac.kr

Min Kyung Park

carrot0412@gmail.com

1 Department of Food Science and Engineering, Ewha Womans University, Seoul 03760, Republic of Korea (transcriptomics), proteins (proteomics), and metabolites (metabolomics). In particular, metabolomics is a comprehensive and quantitative approach for determining dynamic metabolic changes, being a powerful tool that can be efficiently applied in diverse scientific fields, such as food science, biochemistry, and drug discovery (Caboni et al., 2019; Migaud et al., 2020). Metabolites are low-molecularweight organic compounds $(<1000 \mathrm{Da})$ in cellular systems that are related to nutritional values, sensory properties, and the generation of energy for survival. The most prevalent analytical tools for a metabolomics analysis are currently nuclear magnetic resonance (NMR) (Cho et al., 2007; Blakebrough-Hall et al., 2020) and chromatographymass spectrometry (MS) (Gao and Xu, 2015). In particular, MS-based metabolomics analysis has been widely used due to its high selectivity and sensitivity, and potential in identifying metabolites (Dettmer et al., 2007). MS-based instrumental techniques including gas chromatography (GC)-MS, liquid chromatography (LC)-MS, and capillary electrophoresis (CE)-MS, are generally applied to investigate a large range of metabolites.

Table 1 lists the MS-based metabolomics that are actively used as tools for monitoring changes in intra- and extracellular metabolites during fermentation, for evaluating metabolic activities, and the non-target effects of mixed culture, for discriminating geographical origins, and for identifying biomarkers or key components related to functional and organoleptic properties. Alves et al. (2020) suggested how the fingerprinting of beer volatiles varied with the brewing steps. Lee et al. (2014) compared the primary and secondary metabolites profiles of doenjang during its industrial processing, including raw material (soybean), steaming step (1 day), drying (2 days), meju fermentation ( 3,4 , and 17 days), brining (22, 40, and 51 days), and aging ( 81 and 141 days). Metabolic data sets 
Table 1 Applications of metabolomics strategy

\begin{tabular}{|c|c|c|c|c|}
\hline Application fields & Sample & Target compounds & Instrument & $\operatorname{Refs}^{\mathrm{a}}$ \\
\hline \multirow[t]{2}{*}{$\begin{array}{l}\text { Tracking of metabolic changes } \\
\text { during fermentation }\end{array}$} & Deonjang & $\begin{array}{l}\text { Primary metabolites } \\
\text { Secondary metabolites }\end{array}$ & $\begin{array}{l}\text { GC-TOF/MS } \\
\text { UPLC-Q-TOF/MS }\end{array}$ & (Lee et al., 2014) \\
\hline & $\begin{array}{l}\text { Beer } \\
\text { Black tea }\end{array}$ & $\begin{array}{l}\text { Volatile metabolites } \\
\text { Secondary metabolites }\end{array}$ & $\begin{array}{l}\text { GC-MS } \\
\text { HPLC-Q-TOF/MS }\end{array}$ & $\begin{array}{l}\text { (Alves et al., 2020) } \\
\text { (Tan et al., 2016) }\end{array}$ \\
\hline \multirow[t]{2}{*}{ Metabolic activity evaluation } & Deonjang & $\begin{array}{l}\text { Volatile metabolites } \\
\text { Enzymatic activities }\end{array}$ & $\begin{array}{l}\text { GC-MS } \\
\text { Spectrometry }\end{array}$ & (Kum et al., 2015) \\
\hline & Cheese & $\begin{array}{l}\text { Primary metabolites } \\
\text { Secondary metabolites }\end{array}$ & $\begin{array}{l}\text { HPLC } \\
\text { LC-MS }\end{array}$ & (Hagi et al., 2019) \\
\hline $\begin{array}{l}\text { Non-target effects of mixed-culture } \\
\text { Discrimination of origin }\end{array}$ & Cheese & $\begin{array}{l}\text { Primary metabolites } \\
\text { Volatile metabolites }\end{array}$ & $\begin{array}{l}\text { UPLC-Q-TOF/MS } \\
\text { GC-MS }\end{array}$ & (Gu et al., 2020) \\
\hline \multirow{5}{*}{ Discrimination of origin } & Makgeolli & $\begin{array}{l}\text { Primary metabolites } \\
\text { Volatile metabolites }\end{array}$ & $\begin{array}{l}\text { GC-TOF/MS } \\
\text { GC-MS }\end{array}$ & (Son et al., 2018) \\
\hline & Wine & Volatile metabolites & GC-MS & (Englezos et al., 2018) \\
\hline & Deonjang & $\begin{array}{l}\text { Primary metabolites } \\
\text { Secondary metabolites }\end{array}$ & $\begin{array}{l}\text { GC-TOF/MS } \\
\text { LC-ESI/MS }\end{array}$ & (Lee et al., 2017) \\
\hline & Soybeans & $\begin{array}{l}\text { Primary metabolites } \\
\text { Secondary metabolites }\end{array}$ & $\begin{array}{l}\text { GC-TOF/MS } \\
\text { LC-Orbitrap MS }\end{array}$ & (Lee et al., 2019) \\
\hline & Ваїіи & Volatile metabolites & $\mathrm{GC} \times \mathrm{GC}-\mathrm{TOF} / \mathrm{MS}$ & (Song et al., 2020) \\
\hline \multirow[t]{2}{*}{ Finding novel biomarker } & Fermented soybeans & $\begin{array}{l}\text { Primary metabolites } \\
\text { Volatile metabolites }\end{array}$ & $\begin{array}{l}\text { GC-TOF/MS } \\
\text { GC-MS }\end{array}$ & (Park and Kim, 2020) \\
\hline & Goat milk & Primary metabolites & GC-MS & (Scano et al., 2014) \\
\hline
\end{tabular}

${ }^{\mathrm{a}}$ Reference

obtained from GC-time of flight (TOF)/MS and ultra-performance liquid chromatography (UPLC)-TOF/MS indicated the major metabolic changes associated with each process. A metabolomics approach can also be used evaluate the metabolic activity, which is related to the levels of specific metabolites produced. For example, knowledge of the profiles of metabolites produced during fermentation could be useful when attempting to enhance the functional properties of cheese. (Hagi et al., 2019). This could also provide some clues about the effects of mixed cultures, the discrimination of geographical origins, or finding novel biomarkers in fermented foods. Song et al. (2020) successfully determined the geographical origin of baijiu samples using volatile metabolite profiles, while Park and Kim (2020) revealed the microbial-specific metabolites in fermented soybeans using the profiles of primary and volatile metabolites and multivariate statistical analysis.

Metabolomics approaches can be divided into two strategies, 'targeted' or 'non-targeted'. Targeted approaches focus on the quantification and identification of preselected metabolites using references libraries (Gold et al., 2015). In targeted analysis, it can provide high selectivity and sensitivity, while it cannot obtain global analysis of metabolome, because it covers only certain metabolite- specific responses. In contrast to targeted metabolomics, non-targeted approaches deal with information on global metabolic changes and clues, possibly leading to the identification of novel biomarkers (Zhao et al., 2018). In non-target analysis, metabolites are not identified and the spectral data of all potential compounds are not preselected (Tikunov et al., 2005). In general, the data collected by non-target analysis follow four basic steps: deconvolution, alignment, filtering and gap filling (Mastrangelo et al., 2015). Before the identification of metabolites, all data sets need to be evaluated using multivariate statistical analysis. Thus, positive identification of metabolites is priority matter in non-target analysis, because enormous quantity of data and lack of information on data can limit the identification.

The success of an investigation of metabolomics is highly dependent on the overall experimental workflow, including sample collection and data interpretation. When analyzing a sample it is necessary to avoid changing the composition of the metabolites, such as via contamination. Extracting volatile metabolites is particularly difficult due to their sensitivity and instability, and this is performed using solvent-free methods [static and dynamic headspace extraction, solid-phase microextraction (SPME), and stir 
bar sorptive extraction (SBSE)] and solvent based extractions [simultaneous distillation extraction (SDE) and solvent-assisted flavor evaporation (SAFE)]. Solvent-based extraction can require further sample preparation, such as solid phase extraction (SPE), solvent evaporation, and chemical derivatization, in order to optimize them for instrumental analytical techniques.

Metabolomics data sets are commonly very large, and so diverse data-mining analysis techniques such as, principle component analysis (PCA), partial least squares (PLS), PLS- discriminant analysis (PLS-DA), and orthogonal projection to latent structures (OPLS), are often used to extract relevant information. Applying such statistical datamining analysis methods in metabolomics research can identify treatment differences or patterns in large data sets. (Hendriks et al., 2011). They result in the projection of the original data on a lower dimensional space capturing as much as possible of the information in the data, that is, the observed variation (Saccenti et al., 2014).

Several software tools have recently become available for the functional and biological interpretation of metabolic data sets. Some tools can map and display associated metabolic data to provide visual representations of metabolism. (Chagoyen and Pazos, 2012). For example, kyoto encyclopedia of genes and genomes (KEGG)'s pathway browser (https://www.genome.jp/kegg) can provide a global view of the metabolism and a list of the involved pathways (Okuda et al., 2008). Also, MetaboAnalyst is a comprehensive Web-based tool that makes it easy to perform metabolomics data analysis, visualization, and functional interpretation. (Chong et al., 2018). In addition, the open-source R software contains a powerful set of functions for creating graphics, from fairly simple graphs using base graphics commands to highly sophisticated graphs using one of several advanced graphics packages. (Grace and Hudson, 2016). However, despite the many developed interpretation techniques, there remains a need to create new analytical platforms for interpreting metabolic data sets more efficiently and accurately.

\section{Fermentation, dynamic metabolic changes derived from microorganisms}

Fermentation is a metabolic process associated with chemical break-down of macro-molecules (carbohydrates, proteins, and lipids) and the synthesis of micromolecules (e.g. volatile metabolites) by microorganisms. The microbial metabolic processes that occur during fermentation include a series of catabolism and anabolism steps involving the breaking down and synthesis of diverse metabolites. In the primary stage of fermentation, microorganisms grow rapidly on the surfaces of surrounding ingredients and usually make some metabolites associated with the growth phase, such as the synthesis of DNA, RNA, and vitamins, the degradation of macromolecular compounds, (carbohydrates, proteins, and lipids), and the generation of energy (ATP, NADH, and others) for their survival (Franco and Pérez-Díaz, 2013). Various hydrolytic enzymes (e.g. amylase, glucoamylase, protease, and lipase) are also involved in the degradation of macromolecules to small molecules (sugars, amino acids, and fatty acids), which can be precursors for secondary metabolic reactions (Park et al., 2010). When microorganisms are present in the later exponential phase or stationary phase, they can form various secondary metabolites related to the functional or organoleptic properties of fermented foods. In particular, volatile metabolites comprise a chemically diverse group of organic compounds that generally have molecular weights in the range of 50-200 Daltons (Rowan, 2011). These compounds significantly affect the qualities of fermented foods, although they comprise only a small proportion of the total number of metabolites produced by microbes.

Based on relevant previous studies (Chen, 2008; Jo et al., 2011; Ferreira and Guido, 2018; Yu et al., 2014), Fig. 1 presents the main microbial metabolic processes involved in the production of volatile metabolites during fermentation, including glycolysis, Ehrlich pathway (amino acid catabolism), lipoxygenation, oxidation/reduction. Multiple microbial metabolic are linked to each metabolite, but certain volatile metabolites can be generated only from specific ones. (Horak et al., 2019). For example, some intermediate metabolites such as pyruvate and acetyl-CoA are linked to several metabolic processes. Also, sulfurcontaining compounds are specifically derived from sulfurcontaining amino acids. The present study investigated fermentation processes by dividing metabolism into three parts involving carbohydrates, amino acids, and fatty acids.

Carbohydrate metabolism (Fig. 2) provides a carbon source for the formation of volatile metabolites as well as microorganism growth (Mao et al., 2005). It also produces other precursors needed for the formation of volatile metabolites, such as amino acids, fatty acids, and metabolic fuels (NADH, $\mathrm{NAD}^{+}, \mathrm{ATP}$, and etc.) related to other metabolic pathways (Berg et al., 2002). Chemical and biological processes occur simultaneously during fermentation. In particular, the Maillard reaction is a common chemical reaction (non-enzymatic reaction) that occurs between amino acids and reducing sugars during heating. Amadori compounds are formed in the initial step of this reaction, which is followed by a series of rearrangements, dehydrations, and cyclization to produce volatiles such as furans, furfural, and Strecker aldehydes, as well as color in fermented foods. (Golon et al., 2014). 


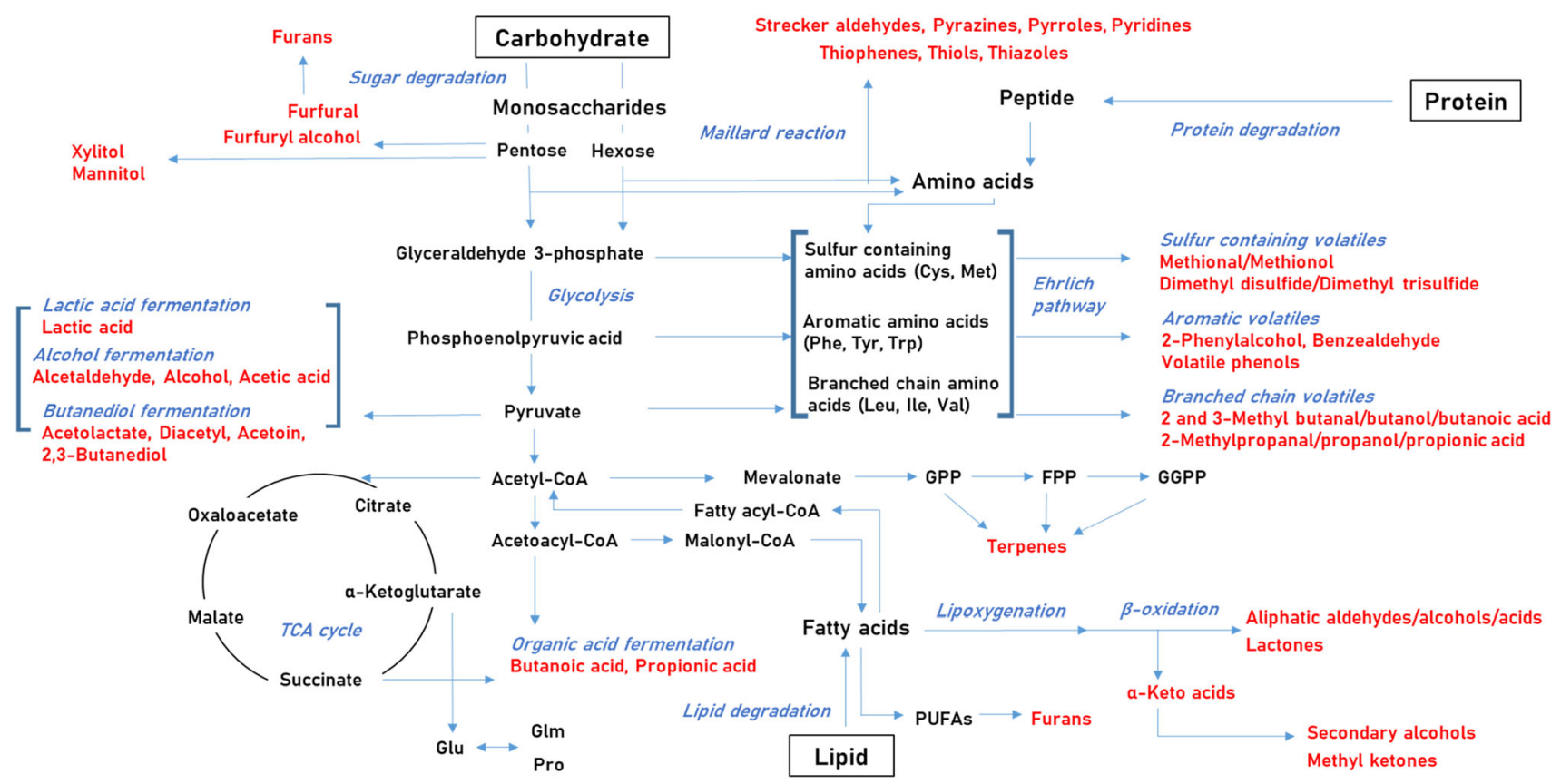

Fig. 1 Main pathways for microorganisms to produce volatile metabolites. All abbreviations were shown as below; cysteine (Cys), methionine (Met), phenylalanine (Phe), tyrosine (Tyr), tryptophan (Trp), leucine (Leu), isoleucine (Ile), valine (Val), geranyl

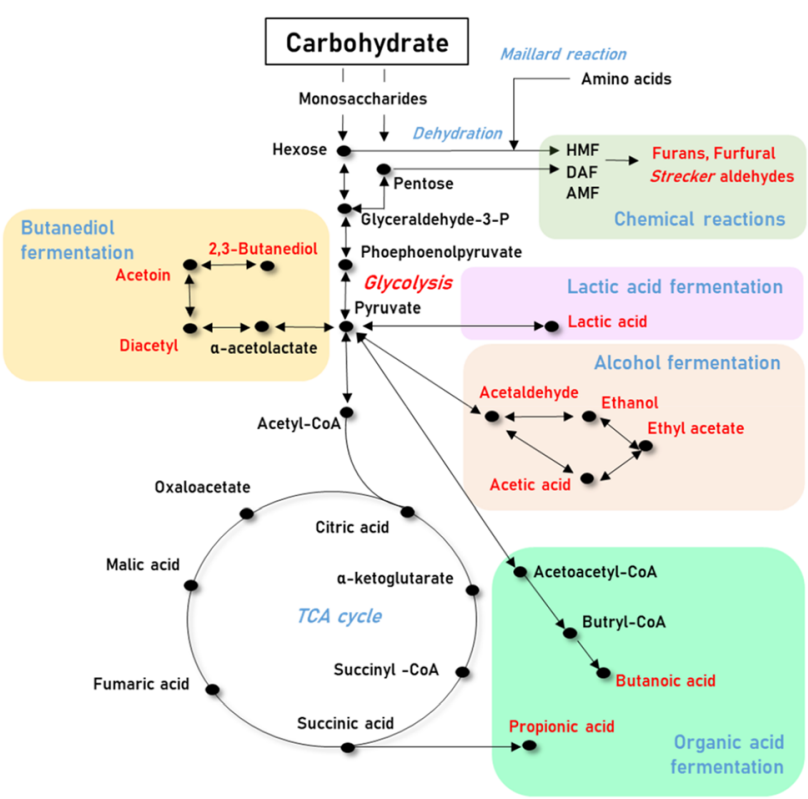

Fig. 2 Overview of the main carbohydrate metabolism related to form volatile metabolites

There are also several important biological pathways involved in the formation of volatile metabolites during the metabolism of carbohydrates, including the fermentation of lactic acid, alcohol, butanediol, and organic acids (butanoic acid and propanoic acid). Lactic acid fermentation is the simplest type of fermentation, which involves the pyrophosphate (GPP), farnesyl pyrophosphate (FPP), geranylgeranyl pyrophosphate (GGPP), polyunsaturated fatty acids (PUFAs), glutamate (Glu), glutamine (Glm), and proline (Pro)

production of lactic acid from pyruvate. Lactic acid can influence the flavor of fermented foods by the production of acids and the lowering of $\mathrm{pH}$, which increases sourness (McFeeters, 2004). The pathways can be divided into the fermentation of homolactic acid (only producing lactic acid) and heterolactic acid fermentation (mainly producing lactic acid, also converting to ethanol and carbon dioxide), with lactic acid bacteria being mainly responsible (Kim et al., 2020). Alcoholic fermentation, in which the main products are ethanol and carbon dioxide, is commonly used for producing alcoholic beverages. (Walker and Stewart, 2016). Acetaldehyde is produced in the initial step by removing the carboxyl group from pyruvate. Ethanol and acetic acid are then formed via the activities of alcohol dehydrogenase and aldehyde dehydrogenase, respectively. (Orywal and Szmitkowski, 2017). Ethyl acetate can also be generated from the combination of ethanol and acetic acid (Jørgensen et al., 2007). 2,3-Butanediol is mainly produced by bacterial species during sugar fermentation, being converted from 2,3-butanedione (diacetyl) and 3-hydroxy2-butanone (acetoin). Pyruvate from glycolysis is condensed into $\alpha$-acetolactate by $\alpha$-acetolactate synthase (ALS), with $\alpha$-acetolactate then being anaerobically transformed into acetoin by $\alpha$-acetolactate decarboxylase (ALDC). Also, $\alpha$-acetolactate can be decarboxylated to diacetyl and also reduced to 2,3-butanediol by 2,3-butanediol dehydrogenase (BDH) (Yang et al., 2017). 
The fermentation of organic acids (e.g., propionic acid and butanoic acid) is mainly observed in the fermentation of certain microorganisms, such as Propionibacterium acidipropionici (Coral et al., 2008), Clostridium, Butyrivibrio, Eubacterium and Fusobacterium species (GonzalezGarcia et al., 2017). The production of butanoic acid starts with the metabolism of glucose to pyruvate via the Embden-Mayerhof-Parnas (EMP) pathway, followed by conversion to acetyl-CoA, and then to acetoacetyl-CoA through a thiolase reaction. The butyryl-CoA produced from acetoacetyl-CoA is then converted into butyryl phosphate by phosphotransbutyrylase, followed by conversion into butanoate by butyrate kinase (Zhu and Yang, 2004). The metabolic pathways related to the production of propanoic acid can be divided into three classes: (i) acrylate and the Wood-Werkman-cycle pathway, (ii) the catabolism of amino acid pathways, and (iii) anabolic pathways associated with the production of biomass precursors from pyruvate or carbon dioxide (Gonzalez-Garcia et al., 2017).

Amino acids are common precursors involved in the production of higher alcohols (fusel alcohols) as well as sulfur- and nitrogen-containing volatile metabolites. The Ehrlich pathway is the main biological catabolism process involving branched-chain amino acids (leucine, isoleucine, and valine), aromatic amino acids (phenylalanine, tyrosine, and tryptophan), and sulfur-containing amino acids (cysteine and methionine), leading to the formation of fusel aldehydes, alcohols, acids, and esters. The Ehrlich pathway comprises three stages: (i) transamination, (ii) decarboxylation, and (iii) oxidation/reduction (Fig. 3). In the initial step, the amino group of amino acids is removed, transferred, or converted into ammonium ions by aminotransferase, and the remaining part participates in further reactions. Key intermediates from the Ehrlich pathway are $\alpha$-keto acids, which are derived either catabolically from exogenous amino acids or result from anabolic pathways involving the biosynthesis of amino acids (Platell et al., 2000). Pyruvate decarboxylase is then converted into the resulting $\alpha$-keto acids to aldehydes by decarboxylation. Finally, aldehydes are converted into acids and alcohols by alcohol dehydrogenase and acid dehydrogenase, respectively (Hazelwood et al., 2008).

Short-chain (comprising fewer than four carbon atoms) aliphatic aldehydes, acids, alcohols, and esters can be generated by the degradation of amino acids via the Ehrlich pathway as well as the degradation of fatty acids. (Hazelwood et al., 2008). This process involves generating various volatile aroma compounds, including carbonyls, higher alcohols, esters, volatile fatty acids, and sulfur compounds, and they are directly dependent on the nitrogen sources that are present during fermentation (Stribny et al., 2015; Torrea et al., 2011). Some volatile metabolites derived from amino acids play important roles in determining the sensory characteristics of fermented foods (Ardö, 2006; Etschmann et al., 2002; Park et al., 2007). In particular, branched-chain amino acids (leucine, isoleucine, and valine) are converted into corresponding aldehydes and acids with characteristic odor descriptions. For example, 3-methylbutanoic acid, which is derived from the degradation of leucine, has a low threshold value and distinctive odor notes (sweaty and cheese-like) and is mainly responsible for the off-odor note of Cheonggukjang (Korean traditional fermented soybeans) (Park et al., 2007). Methanethiol is released directly from the side group of methionine and further converted into other sulfur-containing compounds (Koval, 2005), and it may react with carboxyl acids to produce thioesters, which have boiledcabbage- and cauliflower-like odor notes and are characteristic components of surface-ripened cheeses. (Ardö, 2006). On the other hand, aromatic amino acids (phenylalanine, tyrosine, and tryptophan) are converted into volatile metabolites contributing to flavors, such as roselike, flowers, and bitter almond (e.g. benzaldehyde, 2-phenylethanol, and styrene) as well as chemical, putrid, and faecal flavors (e.g. indole and toluene) (Bertuzzi et al., 2018).

During fermentation, lipids can be hydrolyzed to glycerol and fatty acids by lipase. Glycerol can serve as a fermentation substrate for yeast as well as bacteria species (Wang et al., 2001). Some previous studies have shown that glycerol is also used as a carbon and energy source during carbohydrate metabolism (Himmi et al., 2000). The synthesized fatty acids comprise similar-length and straight carbon chains that have various numbers of carbon atoms $\left(<\mathrm{C}_{6}\right.$, short chain; $\mathrm{C}_{6-12}$, medium chain; $>\mathrm{C}_{14}$, long chain) and different degrees of unsaturation (saturated, mono-unsaturated, and polyunsaturated) (Yu et al., 2014). They are usually degraded by $\beta$-oxidation, which is the breakdown of fatty acids into two carbon segments (acetylCoA) (Campbell et al., 2003). The degradation of fatty acids can generate further various volatile metabolites.

Figure 4 shows various fatty acids derived metabolites, such as fatty acid esters, straight chain aliphatic volatile metabolites $\left(>\mathrm{C}_{6}\right)$, and lactones. Fatty acids-derived volatiles are mainly generated from $\beta$-oxidation or lipoxygenation of fatty acids (Catalá, 2010). Esters contribute to aroma properties such as fruity and flora odor descriptions in most fermented products (Francis and Newton, 2005; Reineccius. 2006), while esters with high molecular weights commonly exhibit fatty and oily aroma notes (Reineccius, 2006). Esters can be produced by the esterification of alcohols with fatty acids (Zhao et al., 2009).

Furans are mainly formed by the oxidative degradation of polyunsaturated fatty acids (Perez and Yaylayan, 2004). Some furan derivatives reportedly have characteristic odor 
Fig. 3 Scheme of Ehrlich pathway and amino acid degradation

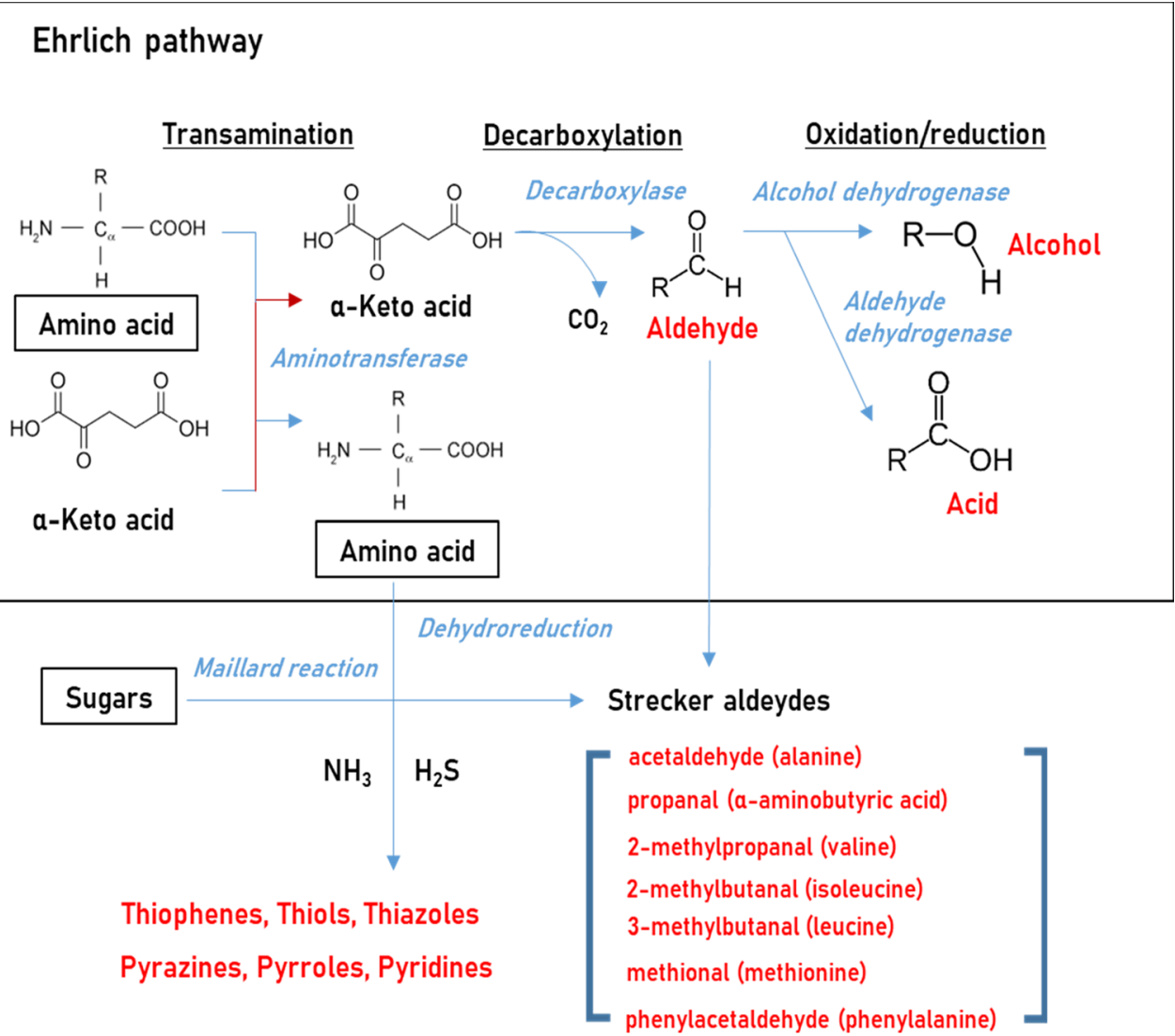

descriptions. For example, furan compounds such as 2-pentylfuran, 2,3-dihydro-benzofuran, and 2-N-octylfuran are characterized by strongly scented, sweet, and (in some cases) burnt odor notes, and are generally considered to be important aromatic compounds that contribute greatly to the flavors of soy sauce (Diez-Simon et al., 2020). Lactones are produced by unsaturated fatty acids such as linoleic acid and oleic acid during fermentation. (Romero-Guido et al., 2011). These lactones have characteristic odor descriptions, such as buttery and peach-like odor notes, and contribute significantly to the aroma of certain fermented alcoholic beverages and dairy products $\mathrm{Zu}$ and Xiao, 2019).

\section{Further omics strategies}

An integrated omics approach has recently been regarded as an useful strategy for evaluating biological and functional properties at multiple scales to detect novel and hidden metabolites or pathways. Integrated omics data include multiple data sets collected using different technologies, such as genome-sequencing and metabolite profiles. Many possible pathways link genotypes and phenotypes, being represented by distinct functional states of cellular components (genes, metabolites, DNA methylation states, and proteins) (Fondi and Liò, 2015). Rossouw et al. (2012) used a multi-omics analysis to investigate the effects of the co-inoculation of commercial yeast (Saccharomyces cerevisiae) as a bacterial starter culture (Oenococcus oeni) in synthetic must. They compared transcriptome and flavor-active metabolite profiles, and some of the differentially expressed genes appeared to respond to chemical changes during fermentation. The upand down-regulated genes were also determined, and the impacts on fermentation kinetics associated with the produced flavors and aromas were explained. Afshari et al. (2020) investigated the condition-specific microbes and metabolites on the surface of cheese using a multi-omics strategy (transcriptomics and metabolomics). They demonstrated that such a strategy represents a highly sensitive and reliable tool for distinguishing between closely related industrial and artisanal cheddar cheeses and brands (Afshari et al., 2020).

In general, the connection between gene expression and produced metabolites is estimated based on hypothesized cause-and-effect relationships. However, this approach can be challenging when interpreting data obtained using different technologies. Fondi and Liò (2015) proposed three main computational challenges: (i) tracking the different molecular components (i.e., genes, molecules, and enzymes) across different data sets and experiments, (ii) 
Fig. 4 Overview of metabolic pathways that lead to production of fatty acids and fatty acidderived metabolites. The abbreviations are shown as below; polyunsaturated fatty acids (PUFAs). fatty acid methyl esters (FAMEs), and fatty acid ethyl esters (FAEEs)

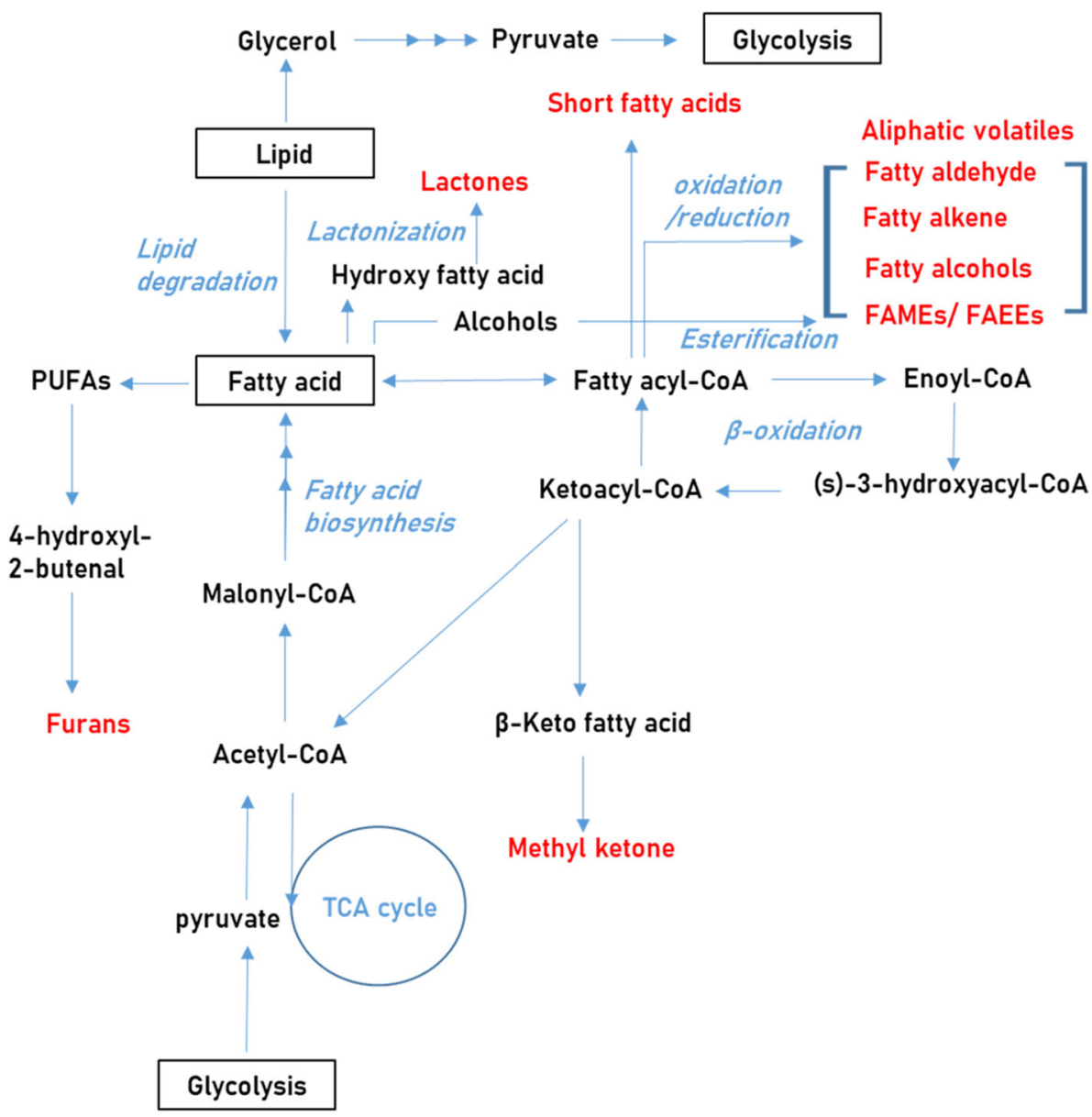

identifying (or developing) reliable data normalization procedures and multi-level data analysis, and (iii) producing an effective visualization of the results. These challenges-from the simple integration of two different data sets to integrating huge numbers of data sets-can be handled for obtaining holistic and comprehensive information about a specific phenomenon or microbial life as a whole. Various databases for metabolic systems are already available for many fermentative microorganisms, and software pipelines have been developed to create models that can predict outcomes when multi-omics data are imputed. (O'Donnell et al., 2020). This situation means that metabolomics now represents a very powerful tool for investigating the complex molecular and biological systems present in fermented foods.

Acknowledgements This work was supported by the National Research Foundation of Korea (NRF) grant funded by the Korea government (MSIT) (No.R1A2015121), and the National Research Foundation of Korea (NRF) and the center for Women In Science, Engineering and Technology (WISET) grant funded by the Ministry of Science and ICT (MSIT) under the program for returners into R\&D (No.WISET 2020-303).

\section{Declarations}

Conflict of interest None of the authors of this study has any financial interest or conflict with industries or parties.

Open Access This article is licensed under a Creative Commons Attribution 4.0 International License, which permits use, sharing, adaptation, distribution and reproduction in any medium or format, as long as you give appropriate credit to the original author(s) and the source, provide a link to the Creative Commons licence, and indicate if changes were made. The images or other third party material in this article are included in the article's Creative Commons licence, unless indicated otherwise in a credit line to the material. If material is not included in the article's Creative Commons licence and your intended use is not permitted by statutory regulation or exceeds the permitted use, you will need to obtain permission directly from the copyright holder. To view a copy of this licence, visit http://creativecommons. org/licenses/by/4.0/.

\section{References}

Afshari R, Pillidge CJ, Read E, Rochfort S, Dias DA, Osborn AM, Gill H. New insights into cheddar cheese microbiota-metabolome relationships revealed by integrative analysis of multiomics data. Scientific Reports. 10: 1-13 (2020)

Alves V, Gonçalves J, Figueira JA, Ornelas LP, Branco RN, Câmara JS, Pereira JA. Beer volatile fingerprinting at different brewing steps. Food Chemistry. 326: 126856 (2020) 
Ardö Y. Flavour formation by amino acid catabolism. Biotechnology Advances. 24: 238-242 (2006)

Berg J, Tymoczko J, Stryer L. Metabolism consist of highly interconnected pathways. Section $30.1 \mathrm{In}$ : Biochemistry 5th ed. W.H. Freeman \& Co.Ltd., New York, USA (2002)

Bertuzzi AS, McSweeney PL, Rea MC, Kilcawley KN. Detection of volatile compounds of cheese and their contribution to the flavor profile of surface-ripened cheese. Comprehensive Reviews in Food Science and Food Safety. 17: 371-390 (2018)

Blakebrough-Hall C, Dona A, D’occhio M, McMeniman J, González L. Diagnosis of bovine respiratory disease in feedlot cattle using blood 1 H NMR metabolomics. Scientific Reports. 10: 1-12 (2020)

Caboni P, Maxia D, Scano P, Addis M, Dedola A, Pes M, Murgia A, Casula M, Profumo A, Pirisi A. A gas chromatography-mass spectrometry untargeted metabolomics approach to discriminate Fiore Sardo cheese produced from raw or thermized ovine milk. Journal of Dairy Science. 102: 5005-5018 (2019)

Campbell JW, Morgan-Kiss RM, E. Cronan Jr J. A new Escherichia coli metabolic competency: growth on fatty acids by a novel anaerobic $\beta$-oxidation pathway. Molecular Microbiology. 47: 793-805 (2003)

Catalá. A synopsis of the process of lipid peroxidation since the discovery of the essential fatty acids. Biochemical and Biophysical Research Communications. 399: 318-323 (2010)

Chagoyen M, Pazos F. Tools for the functional interpretation of metabolomic experiments. Briefings in Bioinformatics. 14: 737-744 (2012)

Chen H. Microbial volatile organic compounds: generation pathways and mass spectrometric detection. China Journal of Biotechnology. 28: 124-133 (2008)

Cho IH, Kim Y-S, Choi H-K. Metabolomic discrimination of different grades of pine-mushroom (Tricholoma matsutake Sing.) using ${ }^{1} \mathrm{H}$ NMR spectrometry and multivariate data analysis. Journal of Pharmaceutical and Biomedical Analalysis. 43: 900-904 (2007)

Chong J, Soufan O, Li C, Caraus I, Li S, Bourque G, Wishart DS, Xia J. MetaboAnalyst 4.0: towards more transparent and integrative metabolomics analysis. Nucleic Acids Research. 46: W486W494 (2018)

Coral J, Karp SG, de Souza Vandenberghe, Luciana Porto, Parada JL, Pandey A, Soccol CR. Batch fermentation model of propionic acid production by Propionibacterium acidipropionici in different carbon sources. Applied Biochemistry and Biotechnology. 151: 333-341 (2008)

Dettmer K, Aronov PA, Hammock BD. Mass spectrometry-based metabolomics. Mass Spectrometry Reviews. 26: 51-78 (2007)

Diez-Simon C, Eichelsheim C, Mumm R, Hall RD. Chemical and sensory characteristics of soy sauce: A review. Journal of Agricultural and Food Chemistry. 68: 11612-11630 (2020)

Englezos V, Rantsiou K, Cravero F, Torchio F, Pollon M, Fracassetti D, Ortiz-Julien A, Gerbi V, Rolle L, Cocolin L. Volatile profile of white wines fermented with sequential inoculation of Starmerella bacillaris and Saccharomyces cerevisiae. Food Chemistry. 257: 350-360 (2018)

Etschmann M, Bluemke W, Sell D, Schrader J. Biotechnological production of 2-phenylethanol. Applied Microbiology and Biotechnology. 59: 1-8 (2002)

Ferreira I, Guido L. Impact of wort amino acids on beer flavour: A review. Fermentation. 4: 23 (2018)

Fondi M, Liò P. Multi-omics and metabolic modelling pipelines: challenges and tools for systems microbiology. Microbiology Research. 171: 52-64 (2015)

Francis I, Newton J. Determining wine aroma from compositional data. Australian Journal of Grape and Wine Research. 11: 114-126 (2005)
Franco W, Pérez-Díaz I. Microbial interactions associated with secondary cucumber fermentation. Journal of Applied Microbiology. 114: 161-172 (2013)

Gao P, Xu G. Mass-spectrometry-based microbial metabolomics: recent developments and applications. Analytical and Bioanalytical Chemistry. 407: 669-680 (2015)

Gold N.D, Gowen C.M, Lussier F-X, Cautha, S.C, Mahadevan R, Martin V.J.J. Metabolic engineering of a tyrosine-overproducing yeast platform using targeted metabolomics. Microbial Cell Factories, 14: 73 (2015)

Golon A, Kropf C, Vockenroth I, Kuhnert N. An investigation of the complexity of Maillard reaction product profiles from the thermal reaction of amino acids with sucrose using high resolution mass spectrometry. Foods. 3: 461-475 (2014)

Gonzalez-Garcia RA, McCubbin T, Navone L, Stowers C, Nielsen LK, Marcellin E. Microbial propionic acid production. Fermentation 3: 21 (2017)

Grace SC, Hudson DA. Processing and visualization of metabolomics data using R. pp 63-94. In: Metabolomics-Fundamentals and Applications. Prasain P (Ed.). IntechOpen limited, London, UK (2016)

Gu Y, Li X, Xiao R, Dudu OE, Yang L, Ma Y. Impact of Lactobacillus paracasei IMC502 in coculture with traditional starters on volatile and non-volatile metabolite profiles in yogurt. Process Biochemistry.. 99: 61-69 (2020)

Hagi T, Nakagawa H, Ohmori H, Sasaki K, Kobayashi M, Narita T, Nomura M. Characterization of unique metabolites in $\gamma$ aminobutyric acid-rich cheese by metabolome analysis using liquid chromatography-mass spectrometry. Journal of Food Biochemistry. 43: e13039 (2019)

Hazelwood LA, Daran JM, van Maris AJ, Pronk JT, Dickinson JR. The Ehrlich pathway for fusel alcohol production: a century of research on Saccharomyces cerevisiae metabolism. Applied and Environmental Microbiology. 74: 2259-2266 (2008)

Hendriks MM, van Eeuwijk FA, Jellema RH, Westerhuis JA, Reijmers TH, Hoefsloot HC, Smilde AK. Data-processing strategies for metabolomics studies. Trends in Analytical Chemistry. 30: 1685-1698 (2011)

Himmi E, Bories A, Boussaid A, Hassani L. Propionic acid fermentation of glycerol and glucose by Propionibacterium acidipropionici and Propionibacterium freudenreichii ssp. shermanii. Applied Microbiology and Biotechnology. 53: 435-440 (2000)

Horak I, Engelbrecht G, van Rensburg PJJ, Claassens S. Microbial metabolomics: essential definitions and the importance of cultivation conditions for utilizing Bacillus species as bionematicides. Journal of Applied Microbiology. 127: 326-343 (2019)

Jo Y-J, Cho IH, Song CK, Shin HW, Kim Y-S. Comparison of fermented soybean paste (Doenjang) prepared by different methods based on profiling of volatile compounds. Journal of Food Science. 76: C368-C379 (2011)

Jørgensen B, Christiansen SE, Thomsen MLD, Christensen $\mathrm{CH}$. Aerobic oxidation of aqueous ethanol using heterogeneous gold catalysts: Efficient routes to acetic acid and ethyl acetate. Journal of Catalysis. 251: 332-337 (2007)

Kim KH, Chun BH, Baek JH, Roh SW, Lee SH, Jeon CO. Genomic and metabolic features of Lactobacillus sakei as revealed by its pan-genome and the metatranscriptome of kimchi fermentation. Food Microbiology. 86: 103341 (2020)

Koval I. Synthesis, structure, and physicochemical characteristics of thiols. Russian Journal of Organic Chemistry. 41: 631-648 (2005)

Kum S-J, Yang S-O, Lee SM, Chang P-S, Choi YH, Lee JJ, Hurh BS, Kim Y-S. Effects of Aspergillus species inoculation and their enzymatic activities on the formation of volatile components in 
fermented soybean paste (doenjang). Journal of Agricultural and Food Chemistry. 63: 1401-1418 (2015)

Lee SY, Lee S, Oh JY, Jeon EJ, Ryu HS, Lee CH. Primary and secondary metabolite profiling of doenjang, a fermented soybean paste during industrial processing. Food Chemistry. 165: 157-166 (2014)

Lee S, Lee S, Singh D, Oh JY, Jeon EJ, Ryu HS, Lee DW, Kim BS, Lee $\mathrm{CH}$. Comparative evaluation of microbial diversity and metabolite profiles in doenjang, a fermented soybean paste, during the two different industrial manufacturing processes. Food Chemistry. 221: 1578-1586 (2017)

Lee EM, Park SJ, Lee J-E, Lee BM, Shin BK, Kang DJ, Choi H-K, Kim Y-S, Lee DY. Highly geographical specificity of metabolomic traits among Korean domestic soybeans (Glycine max). Food Research International. 120: 12-18 (2019)

Mao X, Eksriwong T, Chauvatcharin S, Zhong J. Optimization of carbon source and carbon/nitrogen ratio for cordycepin production by submerged cultivation of medicinal mushroom Cordyceps militaris. Process Biochemistry. 40: 1667-1672 (2005)

Mastrangelo A, Ferrarini A.Rey-Stolle F, García A, Barbas C. From sample treatment to biomarker discovery: A tutorial for untargeted metabolomics based on GC-(EI)-Q-MS. Analytica Chimica Acta 900(5): 21-35

McFeeters R. Fermentation microorganisms and flavor changes in fermented foods. Journal of Food Science. 69: 35-37 (2004)

Migaud M, Gandotra S, Chand HS, Gillespie MN, Thannickal VJ, Langley RJ. Metabolomics to predict antiviral drug efficacy in COVID-19. American Journal of Respiratory Cell and Molecular Biology. 63: 396-398 (2020)

O'Donnell ST, Ross RP, Stanton C. The progress of multi-omics technologies: Determining function in lactic acid bacteria using a systems level approach. Frontiers in Microbiology. 10: 3084 (2020)

Okuda S, Yamada T, Hamajima M, Itoh M, Katayama T, Bork P, Goto S, Kanehisa M. KEGG Atlas mapping for global analysis of metabolic pathways. Nucleic Acids Research. 36: W423W426 (2008)

Orywal K, Szmitkowski M. Alcohol dehydrogenase and aldehyde dehydrogenase in malignant neoplasms. Clinical and Experimental Medicine. 17: 131-139 (2017)

Park MK, Kim Y-S. Comparative metabolic expressions of fermented soybeans according to different microbial starters. Food Chemistry. 305: 125461 (2020)

Park MK, Choi H-K, Kwon D-Y, Kim Y-S. Study of volatile organic acids in freeze-dried Cheonggukjang formed during fermentation using SPME and stable-isotope dilution assay (SIDA). Food Chemistry. 105: 1276-1280 (2007)

Park MK, Cho IH, Lee SR, Choi H-K, Kwon D-Y, Kim Y-S. Metabolite profiling of Cheonggukjang, a fermented soybean paste, during fermentation by gas chromatography-mass spectrometry and principal component analysis. Food Chemistry. 122: 1313-1319 (2010)

Perez Locas C, Yaylayan VA. Origin and mechanistic pathways of formation of the parent furan -A food toxicant. Journal of Agricultural and Food Chemistry. 52: 6830-6836 (2004)

Platell C, Kong SE, McCauley R, Hall JC. Branched-chain amino acids. Journal of Gastroenterology and Hepatology. 15: 706-717 (2000)

Reineccius G. Flavor Chemistry and Technology. Taylor \& Francis, New York, USA. pp. 73-98. (2006)

Romero-Guido C, Belo I, Ta TMN, Cao-Hoang L, Alchihab M, Gomes N, Thonart P, Teixeira JA, Destain J, Waché Y. Biochemistry of lactone formation in yeast and fungi and its utilisation for the production of flavour and fragrance compounds. Applied Microbiology and Biotechnology. 89: 535-547 (2011)
Rowan DD. Volatile metabolites. Metabolites. 1: 41-63 (2011)

Rossouw D, Toit M.D., Bauer F.F. The impact of co-inoculation with Oenococcus oeni on the trancriptome of Saccharomyces cerevisiae and on the flavour-active metabolite profiles during fermentation in synthetic must. Food Microbiology. 29: 121-131 (2012)

Saccenti E, Hoefsloot HC, Smilde AK, Westerhuis JA, Hendriks MM. Reflections on univariate and multivariate analysis of metabolomics data. Metabolomics. 10: 361-374 (2014)

Scano P, Murgia A, Pirisi FM, Caboni P. A gas chromatography-mass spectrometry-based metabolomic approach for the characterization of goat milk compared with cow milk. Journal of Dairy Science. 97: 6057-6066 (2014)

Son EY, Lee SM, Kim MJ, Seo JA, Kim Y-S. Comparison of volatile and non-volatile metabolites in rice wine fermented by Koji inoculated with Saccharomycopsis fibuligera and Aspergillus oryzae. Food Research International. 109: 596-605 (2018)

Song X, Jing S, Zhu L, Ma C, Song T, Wu J, Zhao Q, Zheng F, Zhao $\mathrm{M}$, Chen F. Untargeted and targeted metabolomics strategy for the classification of strong aroma-type baijiu (liquor) according to geographical origin using comprehensive two-dimensional gas chromatography-time-of-flight mass spectrometry. Food Chemistry. 314: 126098 (2020)

Stribny J, Gamero A, Pérez-Torrado R, Querol A. Saccharomyces kudriavzevii and Saccharomyces uvarum differ from Saccharomyces cerevisiae during the production of aroma-active higher alcohols and acetate esters using their amino acidic precursors. International Journal of Food Microbiology. 205: 41-46 (2015)

Tan J, Dai W, Lu M, Lv H, Guo L, Zhang Y, Zhu Y, Peng Q, Lin Z. Study of the dynamic changes in the non-volatile chemical constituents of black tea during fermentation processing by a non-targeted metabolomics approach. Food Research International. 79: 106-113 (2016)

Tikunov Y, Lommen A, de Vos CHR, Verhoeven HA, Bino RJ, Hall RD, Bovy AG. A novel approach for nontargeted data analysis for metabolomics. Large-scale profiling of tomato fruit volatiles. Plant Physiology. 139: 1125-1137 (2005)

Torrea D, Varela C, Ugliano M, Ancin-Azpilicueta C, Francis IL, Henschke PA. Comparison of inorganic and organic nitrogen supplementation of grape juice-Effect on volatile composition and aroma profile of a Chardonnay wine fermented with Saccharomyces cerevisiae yeast. Food Chemistry. 127: 1072-1083 (2011)

Walker G, Stewart G. Saccharomyces cerevisiae in the production of fermented beverages. Beverages. 2: 30 (2016)

Wang Z, Zhuge J, Fang H, Prior BA. Glycerol production by microbial fermentation: a review. Biotechnology Advances. 19: 201-223 (2001)

Yang T, Rao Z, Zhang X, Xu M, Xu Z, Yang S. Metabolic engineering strategies for acetoin and 2, 3-butanediol production: advances and prospects. Critical Review in Biotechnology. 37: 990-1005 (2017)

Yu A, Juwono P, Kurniasih N, Leong SSJ, Chang MW. Production of fatty acid-derived valuable chemicals in synthetic microbes. Frontiers in Bioengineering and Biotechnology. 2: 78 (2014)

Zhao Y, Xu Y, Li J, Fan W, Jiang W. Profile of volatile compounds in 11 brandies by headspace solid-phase microextraction followed by gas chromatography-mass spectrometry. Journal of Food Science. 74: C90-C99 (2009)

Zhao H., Liu Y, Li z, Song Y, Cai X, Liu Y, Zhang T, Yang L, Li L, Gao S, Li Y, Yu C. Identification of essential hypertension biomarkers in human urine by non-targeted metabolomics base on UPLC-Q-TOF/MS. Clinica Chimica Acta. 486: 192-98 (2018)

Zhu J, Xiao Z. Characterization of the key aroma compounds in peach by gas chromatography-olfactometry, quantitative 
measurements and sensory analysis. European Food Research and Technology. 245: 129-141 (2019)

Zhu Y, Yang S. Effect of $\mathrm{pH}$ on metabolic pathway shift in fermentation of xylose by Clostridium tyrobutyricum. Journal of Biotechnology. 110: 143-157 (2004)
Publisher's Note Springer Nature remains neutral with regard to jurisdictional claims in published maps and institutional affiliations. 